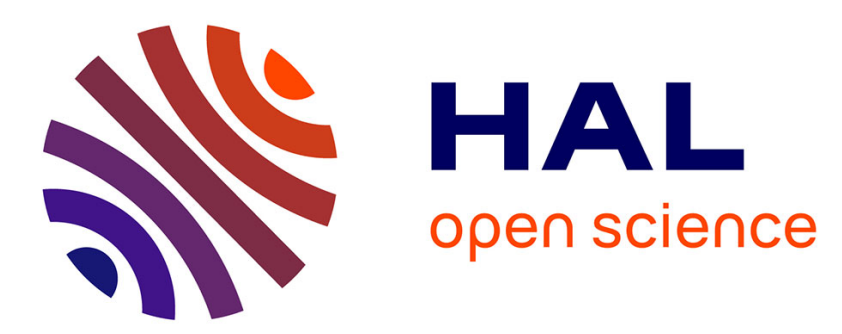

\title{
Les entreprises encyclopédiques catholiques au XIXe siècle: quelques aspects liés à la construction du savoir littéraire
}

\author{
Stéphanie Dord-Crouslé
}

\section{- To cite this version:}

Stéphanie Dord-Crouslé. Les entreprises encyclopédiques catholiques au XIXe siècle: quelques aspects liés à la construction du savoir littéraire. Lise Andries. La construction des savoirs (XVIIIe - XIXe siècles), Presses Universitaires de Lyon, pp.177-196, 2009, Littérature et idéologies. halshs-00391552

\section{HAL Id: halshs-00391552 \\ https://shs.hal.science/halshs-00391552}

Submitted on 20 Aug 2015

HAL is a multi-disciplinary open access archive for the deposit and dissemination of scientific research documents, whether they are published or not. The documents may come from teaching and research institutions in France or abroad, or from public or private research centers.
L'archive ouverte pluridisciplinaire HAL, est destinée au dépôt et à la diffusion de documents scientifiques de niveau recherche, publiés ou non, émanant des établissements d'enseignement et de recherche français ou étrangers, des laboratoires publics ou privés. 
Le texte ci-dessous est la version « auteur » de l'article :

Stéphanie Dord-Crouslé. Les entreprises encyclopédiques catholiques au XIXe siècle : quelques aspects liés à la construction du savoir littéraire. Lise Andries. La construction des savoirs (XVIIIe - XIXe siècles), Presses Universitaires de Lyon, p. 177-196, 2009, Littérature et idéologies.

A été ajoutée la pagination de l'article publié.

[p. 177]

\section{Les entreprises encyclopédiques catholiques au $\mathrm{XIX}^{\mathrm{e}}$ siècle : quelques aspects liés à la construction du savoir littéraire}

La diffusion de la philosophie des Lumières et le cortège de tribulations qui a accompagné la Révolution ont profondément mis à mal la position occupée - et l'influence exercée - par l'Église catholique dans le paysage intellectuel français jusqu'au milieu du XVIII siècle. Pourtant, la première moitié du $\mathrm{XIX}^{\mathrm{e}}$ siècle est marquée par les signes multiples et protéiformes d'une véritable renaissance catholique. Dans le domaine des idées, «l'école catholique et monarchique », issue «de la réaction intellectuelle et morale provoquée par les malheurs inouïs et les crimes étranges sortis de la révolution », selon les termes du critique catholique Alfred Nettement, œuvre pour le «retour solennel à la vérité catholique, donnée pour base à l'ensemble des connaissances humaines, pour solution à tous les problèmes intellectuels et sociaux $»^{1}$.

L'un des hommes qui ont le plus profondément influé sur le développement de ces idées est Lamennais : c'est lui qui, dès le début des années 1820, a mis en avant la notion de « science catholique », notion explicitée en 1829, et, surtout, dans un article du journal L'Avenir en $1831^{2}$. De quoi s'agit-il ? Lamennais et ses amis partent d'un double constat: d'une part, les découvertes scientifiques incessantes, [p. 178] souvent, se contredisent ; d'autre part, la spécialisation croissante des champs scientifiques crée une impression de

\footnotetext{
${ }^{1}$ Alfred Nettement, Histoire de la littérature française sous la Restauration, Paris, Lecoffre, 1853, t. 1, p.175.

${ }^{2}$ Voir Jean-René Derré, Le Renouvellement de la pensée religieuse en France de 1824 à 1834. Essai sur les origines et la signification du mennaisisme, Paris, Klincksieck, 1962.
} 
morcellement. Le sens fait donc partout défaut. Pour pallier ce manque, il faut redonner un sens (catholique) aux faits scientifiques, tout en travaillant le contenu du catholicisme pour l'élever jusqu'au niveau de la science. Seule une réussite conjointe sur ces deux tableaux peut permettre de dépasser le divorce consommé au siècle précédent entre la science et le catholicisme, et de remédier ainsi à l'illégitimité dont est frappée l'Église, depuis l'épisode révolutionnaire, lorsqu'elle se préoccupe des domaines scientifiques ${ }^{3}$.

Une véritable innovation apologétique (la construction de la notion de «science catholique ») et un profond désir de revanche (visant à réparer l'humiliation que les intellectuels catholiques éprouvent à voir le flambeau de la science dorénavant brandi par des savants déistes ou athées) s'unissent donc pour créer les conditions d'un foisonnement intellectuel intense. Tous les moyens sont bons pour réinvestir le champ des savoirs. La presse est largement mise à contribution. Mais c'est à un autre type d'entreprise éditoriale que je voudrais ici m'intéresser, parce qu'il est véritablement au cœur de ce projet de réconciliation de l'Église avec la science et qu'il en souligne la complexité des implications idéologiques : il s'agit des entreprises encyclopédiques catholiques qui fleurissent à cette époque.

Une fois que j'aurai dressé à grands traits le panorama des différentes entreprises pouvant répondre à cette définition, j'essayerai d'exposer les raisons diverses pour lesquelles les notions d'encyclopédie et de catholicisme font alors si bon ménage, ou du moins - rupture de symétrie - pourquoi cette forme générique a alors autant reçu les suffrages des milieux intellectuels catholiques. Enfin, en me concentrant sur quelques aspects, j'essayerai de discerner la place particulière qu'occupe le champ littéraire dans la « science catholique » telle qu'elle est construite, à cette époque, par les encyclopédies catholiques.

[p. 179]

\section{Une floraison d'entreprises diverses, signe d'un profond renouveau idéologique}

«Siècle des dictionnaires », le XIX ${ }^{\mathrm{e}}$ est aussi celui des encyclopédies, et les milieux intellectuels catholiques ne sont pas les moins actifs dans cette production intensive, où l'on peut voir la traduction massive (en nombre de volumes imprimés et en place occupée sur les rayons de bibliothèques) du renouveau spirituel dont j'ai esquissé le tableau dans mon introduction. Si les entreprises encyclopédiques catholiques fleurissent, elles se présentent néanmoins sous des formes éditoriales diverses et ont connu des fortunes variables. Pour simplifier, je distinguerai deux grandes catégories : les entreprises qui reposent sur le principe

\footnotetext{
${ }^{3}$ Sur cette question, voir François Laplanche : «La notion de science catholique : ses origines au début du $\mathrm{XIX}^{\mathrm{e}}$ s. », Revue d'histoire de l'Église de France, t. 74, 1988, p.63-89; et « Du catholicisme comme somme des savoirs à la période romantique », L'entreprise encyclopédique [Littérales, $\mathrm{n}^{\circ} 21$ ], sous la dir. de Jean Bouffartigue et Françoise Mélonio, Nanterre, Centre des sciences de la littérature, Université Paris X, 1997, p.327-340.
} 
de la bibliothèque, c'est-à-dire de la collection d'ouvrages autonomes traitant chacun un aspect particulier du savoir, et les entreprises qui présentent l'ensemble des connaissances sous forme d'articles ordonnés alphabétiquement et placés sous la dépendance d'un titre unique.

En tête de la première catégorie se détache l'entreprise de l'abbé Migne, prêtre généralement connu pour ses Patrologies grecque et latine qui sont aujourd'hui encore, dans la plupart des cas, des outils de référence pour les historiens qui travaillent sur l'Église des premiers siècles. Cependant, ces deux séries d'ouvrages ne sont que la partie émergée (dans notre mémoire collective) d'un iceberg bien plus considérable dont quelques travaux récents ${ }^{4}$ ont juste commencé à esquisser les larges contours. L'abbé Migne a édité une Bibliothèque universelle du clergé et des lä̈ques instruits ou Cours complets sur chaque branche de la science religieuse et humaine, dont les premiers titres ont paru en 1837 et qui comprend au total plus de mille volumes répartis en une petite dizaine de collections ${ }^{5}$.

La conception de cette Bibliothèque universelle répondait à des aspirations diverses. L'abbé Migne faisait d'abord montre d'un réel souci documentaire, suscité par la raréfaction, voire la quasi-disparition, de certains titres du domaine religieux, qui avaient particulièrement souffert de l'épisode révolutionnaire. Il était donc urgent de [p. 180] les remettre à la disposition de tous, et ainsi de sauver cette partie menacée du patrimoine intellectuel de l'Église ${ }^{6}$. Mais diffuser n'est pas tout, encore faut-il le faire à un coût raisonnable pour que l'opération soit vraiment efficace. Aussi la publicité, à laquelle l'éditeur a amplement eu recours, insiste-t-elle sur ce point. L'un des prospectus, glosant le titre latin des Patrologies, décrit le but poursuivi par Migne en ces termes : «reproduire la tradition catholique, universellement quant aux auteurs, intégralement quant aux ouvrages, chronologiquement quant à la marche, uniformément quant au format, économiquement quant au prix $»^{7}$. Pour répondre à cet impératif de bon marché, l'abbé Migne a dû relever un double défi : fabriquer en grande quantité, sans pour autant renoncer à la qualité. Les Ateliers catholiques du Petit-Montrouge se devaient de surpasser l'Imprimerie nationale dans son savoir-faire technique et dans sa capacité de production. La compétition assumée avec les institutions laïques ne se limitait d'ailleurs pas aux questions techniques; elle mettait en cause la légitimité des lieux de

\footnotetext{
${ }^{4}$ Voir La Science catholique : l'"Encyclopédie théologique» de Migne (1844-1873) entre apologétique et vulgarisation, sous la dir. de Claude Langlois et François Laplanche, Paris, Éd. du Cerf (Histoire), 1992 ; et R. Howard Bloch, Le plagiaire de Dieu : la fabuleuse industrie de l'abbé Migne, Paris, Seuil (La librairie du XX siècle), 1996.

${ }^{5}$ Voir le descriptif complet à l'art. «Migne» dans le Dictionnaire de théologie catholique, t. 10, vol. 2, col. 1728-1738.

${ }^{6}$ Dans une lettre adressée à Louis Veuillot du 13 février 1858, Migne « espère bien mourir le prêtre du monde entier qui [...] aura fait le plus de bien [à l'Église] en ressuscitant intégralement sa tradition »; cité par R. Howard Bloch, Le plagiaire de Dieu, p.22.

${ }^{7}$ Archives nationales F18 369 ; cité par R. Howard Bloch, Le plagiaire de Dieu, p.72.
} 
conservation patrimoniale, au premier rang desquels la Bibliothèque royale, nationale ou impériale (selon les époques). En 1864, l'abbé Migne écrivait triomphalement dans les Annales de philosophie chrétienne : «[... la Patrologie est désormais le fondement nécessaire de toute bibliothèque sérieuse. Celle même de la rue de Richelieu ne fournirait pas les éléments nécessaires à former notre Cours tout entier ${ }^{8}$.

Se profile ici la véritable ambition - idéologique et scientifique - de l'entreprise éditoriale de Migne : il s'agit, dans une perspective contre-révolutionnaire latente, de saper les fondements de la science profane qui s'est édifiée sur les idées transmises par les philosophes $\mathrm{du} \mathrm{XVIII}^{\mathrm{e}}$ siècle, en présentant un autre système de référence. L'un des proches de l'abbé Migne, l'abbé Augustin Bonnetty, n'affirme-t-il pas en 1866 que cette « encyclopédie [celle de d'Alembert et de Diderot], comparée aux deux Patrologies de l'abbé Migne, est un pygmée de science et d'utilité $»^{9}$ ? Et l'abbé lui-même, manquant [p. 181] pour le moins de la vertu d'humilité, va jusqu'à s'interroger : «Que sont tous les autres travaux littéraires ! Que sont les Encyclopédies du XVIII et du XIX ${ }^{\mathrm{e}}$ siècle! Que sont toutes les autres œuvres typographiques ! Des jeux d'enfants, dont le plus grand n'est rien auprès du nôtre ${ }^{10}$.

Aujourd'hui, cependant, le caractère encyclopédique de l'entreprise de Migne se lit moins dans ses Patrologies que dans les trois séries d'ouvrages qui ont été successivement publiées entre 1844 et 1873 sous le titre d'Encyclopédie théologique, ou Série de dictionnaires sur toutes les parties de la science religieuse, offrant en français, et par ordre alphabétique, la plus claire, la plus facile, la plus commode, la plus variée et la plus complète des théologies, soit un ensemble de 171 volumes pour une centaine de titres différents. En 1992, un ouvrage collectif $^{11}$ a ouvert le vaste chantier de l'analyse des différents savoirs représentés dans cette encyclopédie. Ont été traités certaines branches des sciences physiques et naturelles, comme la chimie, la botanique, la géologie et la paléontologie, et quelques domaines des sciences humaines comme les enjeux vitalistes de la physiologie, les implications idéologiques de la vulgarisation médicale ou la diversité des écoles de psychologie. Mais, malheureusement, peu d'intérêt a encore été accordé aux titres qui portent sur le domaine littéraire.

Autre projet encyclopédique sous forme de «bibliothèque », celui conçu par Louis Veuillot. Dans quelle mesure le publiciste, qui a pris les rênes du journal L'Univers en 1842 (journal fondé en 1833 par l'abbé Migne, rappelons-le), a-t-il eu le dessein de rivaliser avec

\footnotetext{
${ }^{8}$ Annales de philosophie chrétienne, $5^{\mathrm{e}}$ série, t. 10, vol. 69 (1864), p.84 ; cité par R. Howard Bloch, Le plagiaire de Dieu, p.17.

${ }^{9}$ Ibid., $5^{\mathrm{e}}$ série, t. 14, vol. 76 (1866), p.412-413 ; cité par R. Howard Bloch, Le plagiaire de Dieu, p.17.

${ }^{10}$ Patrologie latine, vol. 218, col. 1 ; cité par R. Howard Bloch, Le plagiaire de Dieu, p.17.

${ }^{11}$ La Science catholique : l'«Encyclopédie théologique » de Migne, op. cit.
} 
l'éditeur pléthorique du Petit-Montrouge? - je l'ignore. En revanche, d'après sa correspondance de l'année 1849, il est certain que Veuillot envisage de publier un ensemble d'une centaine de volumes qui seront réunis sous le titre général de Bibliothèque nouvelle. Ce sera une réplique catholique aux encyclopédistes du XVIII ${ }^{\mathrm{e}}$ siècle, proposant une solution à tous les problèmes de l'époque. Les collaborateurs pressentis font partie du cercle des intimes ${ }^{12}$, et «l'ensemble de l'entreprise est conçu pour ruiner les conceptions des [p. 182] "docteurs, des notaires, des juristes, des rentiers". La devise générale, à imprimer sur chaque page de garde, est : hors de l'Église point de salut $»^{13}$. Ce projet, pour des raisons aujourd'hui obscures, n'a pas eu le développement escompté. Dans le catalogue de la BnF, seuls cinq ouvrages, publiés entre 1850 et 1851, sont rattachés à la série intitulée : «Bibliothèque nouvelle. Religion, histoire, sciences, littérature, par une société d'écrivains catholiques, sous la direction de M. Louis Veuillot $»^{14}$. Ce succès mitigé n'en est pas moins une nouvelle preuve de l'ambition catholique de reconquête du savoir qui caractérise cette période, dans une sorte de croisade, limitée au domaine intellectuel, qui s'exprime plus clairement encore sous la forme de deux «véritables » encyclopédies ${ }^{15}$.

En effet, 1836 n'est pas seulement «l'an I de l'ère médiatique » ${ }^{16}$, c'est aussi un millésime particulièrement faste pour la production encyclopédique catholique : paraissent, coup sur coup, cette année-là, les premières livraisons de l'Encyclopédie catholique et de l'Encyclopédie du XIX siècle. L'histoire éditoriale de la première, l'Encyclopédie catholique, est relativement simple, bien que les débuts aient été quelque peu chaotiques : un procès a brutalement interrompu sa parution ${ }^{17}$, et la publication n'a repris qu'en 1839 , avec une [p. 183]

\footnotetext{
${ }^{12}$ Melchior Du Lac sera en charge de la doctrine, Théodore Foisset se consacrera au christianisme primitif, Donoso Cortès s'occupera du protestantisme et du socialisme, Guéranger de l'Inquisition, Roux-Lavergne de l'histoire, un ami prêtre de Satan, et Veuillot lui-même des fêtes et cérémonies de l'Église.

${ }^{13}$ Austin Gough, Paris et Rome. Les catholiques français et le pape au XIX siècle, Paris, Éd. de l'Atelier (Églises / Sociétés), 1996, p.177.

${ }^{14}$ I- Ch. de Bremond, Traité élémentaire d'astronomie (1851) ; II- Donoso Cortes, Essai sur le catholicisme, le libéralisme et le socialisme (1851) ; III- Melchior Du Lac, L'Église et l'État (2 vol., 1850-1851) ; IV- Don Pitra, La Hollande catholique (1850); V- Roux-Lavergne, La philosophie de l'histoire (1850).

${ }^{15}$ On trouve aussi au catalogue de la BnF des ouvrages isolés dont on ne sait s'ils ont fait partie de séries plus importantes mais qui participent en tout cas eux aussi de cette ambition catholique polyvalente à l'égard des savoirs. Voir par exemple : L'Encyclopédie catholique et populaire, sous la dir. de M. Dupray de La Mahérie, Paris, Librairie parisienne, 1864 ; L'Encyclopédie catholique, ou Démonstrations dogmatiques, philosophiques et historiques de la doctrine chrétienne, par H. Dorgan, Paris, Dentu, 1845 ; ou encore Le Manuel de tout le monde, ou Petite encyclopédie catholique des connaissances les plus usuelles et les plus pratiques, publié par le "Moniteur des villes et des campagnes", Paris, 20 rue Cassette, 1835.

16 Alain Vaillant et Marie-Ève Thérenty, 1836, l'an 1 de l'ère médiatique. Étude littéraire et historique du journal «La Presse » d'Émile de Girardin, Paris, Nouveau monde éd., 2001.

${ }^{17}$ Encyclopédie catholique. Répertoire universel et raisonné des sciences, des lettres, des arts et des métiers; formant une bibliothèque universelle, et comprenant... tous les dictionnaires spéciaux. Publiée par la société de l'Encyclopédie catholique, sous la direction de MM. le V. Walsh et Max Raoul, et d'un comité d'orthodoxie..., Paris, 5, rue de Mènars, 1836, 4 t. en 2 vol. in- $8^{\circ}$.
} 
direction éditoriale partiellement remaniée ${ }^{18}$. L'entreprise, achevée en 1848, comporte au total 18 volumes in-quarto et a été complétée en 1859 par trois volumes de supplément ${ }^{19}$. L'histoire éditoriale de l'Encyclopédie du XIX siècle est plus longue et plus complexe. Lancée en 1836, l'entreprise, achevée en 1842 , compte alors 25 volumes in-octavo ${ }^{20}$. Dès 1842 , une seconde édition, elle aussi en 25 volumes, est commercialisée ; elle est complétée d'abord, en 1853, par un volume de supplément (A-Z) accompagné d'un volume de «Table méthodique », puis, en 1859, par un nouveau volume de supplément (A-Z); elle comporte donc 28 volumes au total. En 1867 paraît une nouvelle édition, remaniée, de l'Encyclopédie du XIX siècle : 25 volumes auxquels s'ajoutent progressivement des volumes d'Annuaires sur lesquels je reviendrai plus tard. Une quatrième édition, en 70 volumes, voit le jour en 1876 ; et c'est en 1883 que paraît l'ultime version de l'œuvre, en 75 volumes ${ }^{21}$.

La somme de tous les volumes évoqués dans ce panorama, pourtant rapidement dressé, est impressionnante. Cet aspect strictement [p.184] quantitatif est le signe indéniable de l'importance capitale que les milieux intellectuels catholiques ont accordée, dans cette première moitié du $\mathrm{XIX}^{\mathrm{e}}$ siècle, au projet de réinvestir les savoirs. Mais pourquoi cette volonté de reconquête des domaines scientifiques a-t-elle si volontiers épousé la forme encyclopédique, de préférence à toute autre ? Pour répondre à cette question, j’ai choisi de réduire ce corpus foisonnant aux deux seuls ouvrages de forme encyclopédique stricto sensu : l'Encyclopédie catholique et l'Encyclopédie du XIX siècle.

\section{Forme encyclopédique et savoirs catholiques}

Si l'ambition de réinvestissement des savoirs par les milieux intellectuels catholiques passe par une large utilisation de la forme encyclopédique, c'est d'abord parce qu'il y a une

\footnotetext{
${ }^{18}$ Encyclopédie catholique. Répertoire universel et raisonné des sciences, des lettres, des arts et des métiers, formant une bibliothèque universelle, publiée par la société de l'Encyclopédie catholique, sous la dir. de M. l'abbé Glaire..., de M. le V ${ }^{\text {te }}$ Walsh, et d'un comité d'orthodoxie, Paris, Parent-Desbarres, 1839-1848.

19 Supplément à l'Encyclopédie catholique, publié sous la dir. de M. Chantrel, Licencié ès-lettres, ancien professeur de Rhétorique, membre de plusieurs Sociétés savantes, etc., etc. et de M. l'abbé Orse, Aumônier de l'institution des frères des écoles chrétiennes, fondateur et directeur de la bibliothèque de la famille, etc., avec la collaboration d'hommes spéciaux dans les lettres, les sciences et les arts, contenant : Les Progrès nouveaux, les Découvertes récentes dans les Sciences, les Lettres et les Arts ; la Biographie des hommes illustres morts depuis la publication de l'Encyclopédie catholique jusqu'à nos jours, etc. Ce Supplément est le complément indispensable à l'Encyclopédie catholique, publiée en $18 \mathrm{vol}$. in- $4^{\circ}$, sous la direction de M. l'abbé Glaire et de M. le vicomte Walsh, Paris, Parent-Desbarres, [1859].

${ }^{20}$ Encyclopédie du XIX ${ }^{e}$ siècle. Répertoire universel des sciences, des lettres et des arts avec la biographie de tous les hommes célèbres [sous la dir. d'Ange de Saint-Priest], Paris, Au bureau de l'Encyclopédie du XIX ${ }^{e}$ siècle, 1836-1843. En outre, un volume, séparé, se présente comme une introduction à l'ouvrage : Pierre-Sébastien Laurentie, Théorie catholique des sciences, Paris, Au bureau de l'Encyclopédie du XIX siècle, 1836, 92 p.

${ }^{21}$ Les volumes 49-74 sont des volumes de «Complément », par tranches chronologiques, de 1859-1860 (vol. 4950) à 1880 (vol. 74). Le volume 75 est pour sa part entièrement consacré aux « Classifications » pour les volumes 49-70 et 71-74.
} 
harmonie profonde, une consonance évidente, entre l'idéologie religieuse catholique, c'est-àdire universelle, et la forme livresque qui a pour ambition d'exposer la totalité des connaissances humaines d'une époque, à savoir l'encyclopédie.

Outre les traits communs à toute entreprise encyclopédique, quelle que soit son orientation doctrinale ou son identité idéologique (renfermer une bibliothèque complète en un seul ouvrage ${ }^{22}$; ouvrir l'esprit du lecteur ${ }^{23}$; s'en tenir à la vulgarisation sans prétendre à l'érudition ${ }^{24}$ ), l'encyclopédie catholique présente la particularité de proposer une forme propice à l'exposition d'une conception [p. 185] rigoureusement organisée et cohérente du monde, et des rapports de chaque homme avec ce qui l'entoure, en fonction d'un point de vue singulier, évidemment théo-centrique ${ }^{25}$. En effet, tous les domaines du savoir y sont agencés en un harmonieux concert duquel toute note discordante est bannie, comme le souligne l'Encyclopédie catholique en utilisant une métaphore visuelle :

une encyclopédie est, si l'on veut, un lien naturel entre toutes les sciences, destiné à réunir dans un foyer commun les rayons de lumière qui émanent de chacune, afin de produire cette clarté qui est comme le jour moral de l'humanité entière dont elle aide et féconde toutes les entreprises ${ }^{26}$.

Si tous les savoirs se mettent à graviter autour d'un centre lumineux, c'est bien parce que le morcellement ou la contradiction ne sont pas de mise au sein de la science catholique :

Cette sphère de la science universelle, cette concordance harmonique de toutes les fractions du savoir humain devrait être, par sa nature même, synthétique. Partant de l'absolu, c'est-à-dire de Dieu, comme du foyer central et unique, elle devrait rayonner dans toutes les directions, indiquant tous les enchaînements, révélant tous les mystères et chassant toutes les obscurités ${ }^{27}$.

\footnotetext{
${ }^{22}$ Ainsi, la préface du Supplément de l'Encyclopédie catholique s'extasie devant les incommensurables richesses que contiennent les dix-huit volumes in-quarto parus entre 1839 et 1848, et vante la «merveilleuse machine qui répond à toutes les questions de l'agriculteur, du naturaliste, du grammairien, du chimiste, de l'historien, de l'industriel, du théologien, de l'artiste ou du simple curieux, et qui leur donne à tous une solution aux difficultés qu'ils ont rencontrées ou les indications qu'ils cherchent» (t. 1, p.I). Car l'encyclopédie se doit d'être pratique et de «tenir lieu des ouvrages spéciaux que l'on n'a pas sous la main ou qu'il serait trop long et trop pénible de consulter » (Encyclopédie catholique, art. Encyclopédie, t. 11, p.175).

${ }^{23}$ Renseigner promptement et à tout coup n'est pas suffisant, il faut aussi que l'encyclopédie ouvre l'esprit du lecteur et remplisse le rôle incitatif d'un aiguillon : «plaçant une foule d'idée et de faits à la portée d'une foule d'hommes qui n'y songeaient point, qui sans cela peut-être n'en auraient jamais entendu parler, [elle doit faire] pénétrer partout et arriver, pour ainsi dire, de toutes parts cette provocation dont notre intelligence a besoin » (ibid.).

${ }^{24}$ «Ce n'est [...] point à former des savants que l'on doit aspirer ; il ne faut prétendre qu'à donner une idée exacte de toutes les sciences, qu'à réunir toutes les notions usuelles et préliminaires aux études sérieuses et suivies » (ibid.).

25 «Ainsi l'encyclopédie n'est point la science dans son enchaînement, mais c'est plutôt l'ensemble, et pour ainsi dire l'enchaînement de toutes les notions qui doivent entrer dans l'éducation d'un homme avide de connaître tout ce qui concerne l'homme; c'est-à-dire de bien se pénétrer de tout ce qui relie l'homme à Dieu, de bien comprendre sa position individuelle et sa position sociale, ses rapports avec la nature qui l'environne, avec les générations qui ont précédé la sienne, avec le monde idéal avec lequel il se sent lié » (ibid., p.174).

${ }^{26}$ Art. Encyclopédie, t. 11, p.174.

${ }^{27}$ Encyclopédie du XIX ${ }^{e}$ siècle, art. Encyclopédie, t. 11, 1850 (2 éd.), p.535.
} 
Il s'agit cependant là d'un programme que Dieu seul, le véritable encyclopédiste, serait en mesure d'accomplir complètement. La finitude humaine implique la nécessaire imperfection de la production encyclopédique et protège l'homme de la présomption d'omniscience, tandis que, symétriquement, l'universalité des domaines envisagés par la forme encyclopédique se doit de stimuler un sain appétit de connaissance en gardant le fidèle de l'écueil d'un scepticisme « rebelle à la voix de la vérité » ${ }^{28}$.

[p. 186] Loin d'atomiser les savoirs ${ }^{29}$, une encyclopédie catholique les recompose et fonde leur cohérence. En ouverture de l'Encyclopédie catholique, Édouard Alletz propose une classification des connaissances humaines d'après l'Écriture sainte, accompagnée d'un tableau des progrès généraux de l'esprit humain ${ }^{30}$, et développe la thèse selon laquelle « les progrès de l'esprit humain ne peuvent plus être séparés du triomphe des croyances chrétiennes ${ }^{31}$. Car le projet encyclopédique catholique est indissociable d'une relecture de l'histoire qui réconcilie les événements et le sens. Dans sa Théorie catholique des sciences, qui sert d'introduction à l'Encyclopédie du XIX siècle, Pierre-Sébastien Laurentie vise le même but.

Enfin, dernière raison, mais non la moindre, pour laquelle la forme encyclopédique a été privilégiée dans ce processus de reconquête des savoirs, c'est évidemment le désir pressant des milieux intellectuels catholiques de restituer à la pensée religieuse un genre intimement et presque exclusivement associé, depuis la fin du siècle précédent, au combat contre l'Église mené par Diderot et d'Alembert dans leur ouvrage ${ }^{32}$. Il était aussi urgent de permettre aux fidèles de trouver une réponse à leurs divers questionnements scientifiques sans qu'ils aient besoin, pour cela, de recourir à un ouvrage mis à l'Index. C'est pourquoi il ne faut pas se laisser tromper par la déclaration d'apparence irénique qui ouvre l'Encyclopédie catholique. L'un de ses textes liminaires, sous-titré : «Jugement sur l'Encyclopédie du dix-huitième siècle », insiste sur l'importance des avancées opérées par l'ouvrage de Diderot et d'Alembert, qu'il ne s'agirait surtout pas de récuser. Elle n'aurait quant à elle pour dessein que de «relever, à côté des vérités que les encyclopédistes du dernier siècle ont proclamées, celles

\footnotetext{
${ }^{28}$ Encyclopédie catholique, art. Encyclopédie, t. 11, p.175.

${ }^{29}$ L'Encyclopédie du XIX ${ }^{e}$ siècle déplore ainsi «ce fractionnement excessif dont les encyclopédies de Diderot et de ses amis ont fourni un exemple notable » (art. Encyclopédie, t. 11, p.537).

${ }^{30}$ Cette introduction, augmentée et refondue, a été republiée sous le titre : Génie du XIX ${ }^{e}$ siècle, ou Esquisse des progrès de l'esprit humain, depuis 1800 jusqu'à nos jours, Paris, Paulin, 1842-1843.

${ }^{31}$ Génie du XIX $X^{e}$ siècle, p.III.

${ }^{32}$ L'Encyclopédie catholique avait pour «but hautement proclamé d'élever en l'honneur de la religion un monument qui fît oublier celui que la philosophie incrédule s'était érigé à elle-même dans le siècle précédent », et d'offrir à l'Église « un ouvrage digne de sa majesté séculaire, digne de la vérité dont elle est la dispensatrice à travers les âges et par tout l'univers » (Supplément, Préface de J. Chantrel, t. 1, p.I).
} 
qu'ils ont renversées et méconnues ». Le projet semble [p. 187] donc être celui d'une réorganisation globale pacifique, sur des bases paritaires :

L'œuvre du XIX ${ }^{\mathrm{e}}$ siècle est de constater l'existence des principes qu'on avait cru un moment détruits, parce qu'ils étaient cachés sous les ruines, et de les replacer auprès des principes qui les attendent. Notre Encyclopédie n'est donc pas une réaction contre une réaction, c'est la conciliation des différents ordres de vérités qui avaient paru incompatibles au siècle précédent, parce qu'ils sont distincts, le rapprochement des méthodes indépendantes sans être inconciliables, et par conséquent la fin des exclusions injustes ${ }^{33}$.

Mais d'autres textes, bien plus nombreux et au nombre desquels se trouve déjà le prospectus ${ }^{34}$ de la première édition de l'Encyclopédie catholique, battent en brèche cette affectation de concorde et révèlent au contraire la pensée profonde qui sous-tend toutes ces entreprises catholiques : l'Encyclopédie de Diderot et d'Alembert, voilà l'ennemi ! Le procès de l'ouvrage y est en effet mené sur le terrain scientifique et sur le plan idéologique : on lui reproche à la fois de «fourmille[r] de disparités, d'incohérences, de mutilations qui ont allumé plus d'une fois l'indignation d'un de ses principaux fondateurs, de Diderot lui-même », et «d'être devenue, dans les mains qui l'ont écrite, sinon une arme de destruction, du moins un instrument de perturbation sociale $»^{35}$.

[p. 188] Ce combat contre le grand œuvre des Lumières est commun aux deux encyclopédies catholiques qui nous occupent, de même que de leur étude parait se dégager un consensus sur l'utilité et surtout la nécessité de baliser et de construire le champ des savoirs catholiques par cette forme privilégiée de l'encyclopédie. Mais alors, pourquoi deux entreprises exactement contemporaines, donc de facto concurrentes ? Se diviser, c'était amoindrir les chances de parvenir au but... L'esprit de chapelle n'est certainement pas

\footnotetext{
${ }^{33}$ Encyclopédie catholique, «Avant-propos - Jugement sur l'Encyclopédie du dix-huitième siècle », t. 1, p.VIIVIII.

${ }^{34}$ «Catholiques! voici l'arche d'alliance entre la religion de Dieu et la science de l'homme ; voici le livre destiné à détruire jusqu'aux derniers vestiges des doctrines anti-sociales et impies du dernier siècle, la seule vengeance qu'il soit digne de vous de tirer de tous les maux qu'elles vous ont faits ; voici votre réponse à ceux qui vous accusent de ne pas comprendre et de ne pas aimer la science et les arts »; cité par Claude Bénichou, «L'ignorance des savoirs », La Science catholique..., p.102.

${ }^{35}$ Encyclopédie du XIX $x^{e}$ siècle, Avertissement de la première édition, par le comte de Saint-Priest, cité par Claude Bénichou, "L'ignorance des savoirs », La Science catholique..., p.99. Voir aussi l'art. Encyclopédie, t. 11, p.537-538 : «Deux idées présidèrent à la création de cette œuvre ; englober toutes les connaissances humaines, les concentrer dans un grand travail ; et s'en servir comme d'un bélier pour réduire en poudre et détruire ce que l'ancienne société chrétienne avait fait et construit. Il y avait donc à la fois dans cette gigantesque entreprise, qui restera comme un fait important de notre histoire, une conservation et une destruction. Comme arme d'attaque, elle a réussi ; tout en s'annihilant elle-même, elle a fait crouler les antiques murailles d'une société dégénérée. En vain chercherait-on, dans un recueil auquel les plus grands esprits, les plus grands écrivains, les plus spirituels penseurs de l'époque ont coopéré, des renseignements certains et des lumières utiles. L'unité de plan manque à cet ouvrage, qui a vieilli comme l'esprit de doute et de superficielle ironie qui fait se heurter entre elles les diverses parties d'une œuvre, souvent remaniées, toujours incomplètes. Si l'on contemple aujourd'hui ce grand arsenal des destructeurs de la vieille société dépravée, on n’y trouvera plus que des armes brillantes et hors d'usage, dont des mains hardies se sont servies avec un incroyable acharnement, une tactique et une habileté singulières. »
} 
étranger à cette existence parallèle ${ }^{36}$, tout comme des configurations sociales et intellectuelles, fondées sur des affinités et des désaccords que je n'ai pas encore pu tous clairement mettre au jour (mais j'y reviendrai à la fin de cet exposé), peuvent avoir eu leur importance. En tout cas, des lignes de rupture se dessinent nettement qui permettent sinon d'identifier les causes, du moins de différencier assez nettement les deux entreprises tant dans leur présentation de certains champs du savoir, que dans l'évolution, et l'éventuel infléchissement, de leur projet éditorial. Le domaine que je vais maintenant privilégier est celui de la littérature.

[p. 189]

\section{La « science littéraire » au sein de la « science catholique » : consensus et ruptures}

En effet, quelle est donc la place du champ littéraire dans cette « science catholique » que promeuvent nos deux encyclopédies ? Dans les deux cas, c'est un élément clairement intégré au système. L'Encyclopédie catholique définit la littérature comme «la science qui comprend la grammaire, l'éloquence et la poésie, et qu'on appelle autrement belles-lettres $»^{37}$. L'article « LETTRES (BELLES-) » confirme et développe les liens :

Nous entendons par ce mot, auquel il conviendrait de substituer celui de science littéraire, tout ce qui se rapporte aux opérations de l'esprit appliquées aux choses de l'imagination, telles que la poésie, l'éloquence et l'histoire. [...] — Le mot science, pris dans un sens absolu, signifie connaissance des choses, soit naturelles, soit intellectuelles, fondée sur des principes qui sont le résultat des faits manifestés par l'expérience, ou de longues et profondes observations ; en d'autres termes, c'est la connaissance de tout ce qui concerne les opérations de l'esprit appliquées aux objets physiques ou métaphysiques, ou aux choses qui sont hors de nous. Sous ce dernier rapport, les belles-lettres sont une partie importante de la science $\left(\mathrm{V}\right.$. SCIENCE) $\gg^{38}$.

J'aurais pu envisager la manière dont ces articles résistent, pour certains, ou, au contraire, prennent acte des reconfigurations et des nouveaux partages entre littérature, rhétorique et éloquence qui se font jour entre 1750 et $1848^{39}$. Mais j'ai préféré m'arrêter sur certains aspects qui semblent constituer un socle de références communes pour la construction d'une « science

\footnotetext{
${ }^{36}$ Existence parallèle, en effet, puisque chaque entreprise ne prend que très rarement en compte l'existence de sa consœur en dépit de la parution échelonnée et intriquée de leurs différentes éditions. Dans le panorama du genre que dresse l'article Encyclopédie, l'Encyclopédie catholique mentionne très brièvement l'ouvrage directement concurrent : "Quant à l'Encyclopédie du XIX $X^{e}$ siècle, sa marche semble être aussi bien lente et bien embarrassée et quoiqu'elle publie à la fois les premières et les dernières lettres de l'alphabet, nous ne pouvons en aucune façon préjuger quel sera son étendue et son plan » (p.177). Quant à la préface de son Supplément, elle n'en fait plus état lorsqu'elle passe en revue les différentes encyclopédies du temps ! L'Encyclopédie du XIX siècle n'est pas plus diserte : il faut attendre la préface à la troisième édition pour trouver mention (lapidaire) de l'entreprise adverse : «L'Encyclopédie catholique, et l'Encyclopédie des gens du monde, qu'on a souvent appelée l'encyclopédie protestante, n'ont eu l'une et l'autre qu'une édition, terminée avant 1848. » Autre phénomène curieux, l'absence de toute mention de l'Encyclopédie du XIX siècle dans la préface de Pierre Larousse à son Grand Dictionnaire universel en 1865, alors que l'Encyclopédie catholique y est convoquée...

${ }^{37}$ Art. Littérature, t. 13, p.649.

${ }^{38}$ Art. Lettres (Belles-), ibid., p.578.

${ }^{39}$ Voir ici même la contribution de Corinne Saminadayar-Perrin, p.87-111.
} 
littéraire » catholique afin de pouvoir mieux, par la suite, isoler ce qui fait la spécificité de chacune des deux encyclopédies.

Conformément aux idées développées par Bonald sur la littérature comme expression de la société, l'Encyclopédie catholique et l'Encyclopédie du XIX siècle accordent toutes deux une grande importance aux répercussions morales et sociales du développement de certaines formes littéraires contemporaines, au premier rang desquelles le roman, surtout quand il est diffusé sous la forme de feuilletons. L'article que chacune d'entre elles consacre au genre romanesque se termine par une ferme dénonciation du danger que présentent ces œuvres, en particulier lorsqu'elles tombent entre les [p. 190] mains de femmes ou, pire encore, de jeunes filles. L'Encyclopédie du XIX $X^{e}$ siècle s'inquiète des conséquences à long terme, mais en reste à la docte dénonciation : « Il y a là pour l'avenir un danger immense qu'on ne conjurera pas en se contentant de déclarer le roman une lecture dangereuse et frivole, comme on le fait depuis si longtemps sans avoir pu, non pas détruire le mal, mais sans être même parvenu à en arrêter les progrès ${ }^{40}$; tandis que l'Encyclopédie catholique s'adresse directement à son lecteur en des termes pour le moins propres à l'émouvoir : en permettant à une femme de lire les Mystères de Paris :

vous la mêlerez, par la pensée, à la honteuse cohue qui suit le char de la reine Bacchanal; ou bien vous la ferez assister à l'immonde agonie du notaire Ferrand, se tordant dans les convulsions de sa rage lubrique, et mourant dans les fureurs de cette maladie hideuse qu'on ne saurait nommer sans souiller sa voix et salir les oreilles de ceux qui nous entendent; et en sortant de cette lecture, les yeux encore pleins de ces images abominables, vous lui donnerez à former le cœur et l'esprit de votre fille ! Taisez-vous ! vous ne me faites plus pitié, vous me faites horreur $!^{41}$

Cependant, si jusqu'ici les deux entreprises encyclopédiques paraissent n'apporter que de légères nuances à l'expression d'une même idéologie, il y a en réalité entre elles une ligne de fracture. Dans son article «Roman », l'Encyclopédie du XIX siècle commence par dresser un panorama large et complet du genre, en adoptant une démarche chronologique, depuis les origines (le roman dans la littérature antique) jusqu'à une rapide évocation de la tendance réaliste sous l'espèce des romans de Scott. L'auteur de l'article s'arrête alors : «Nous ne pousserons pas plus loin cette rapide revue des romans; les contemporains nous mèneraient trop loin, et nous sommes trop près d'eux d'ailleurs pour en porter un jugement complètement impartial ». Indépendamment des considérations morales que j'ai précédemment évoquées, l'article paraît finalement peu engagé, surtout soucieux d'opérer une revue exhaustive exposant méthodiquement les développements et les mutations propres à chaque époque et à chaque pays, sans empiéter sur la frange instable des débats contemporains clairement rejetés

\footnotetext{
${ }^{40}$ Art. Roman, t. 21, 1846 ( $2^{\mathrm{e}}$ éd.), p.477 ; et t. 20, 1867 ( $3^{\mathrm{e}}$ éd.), p.477.

${ }^{41}$ Art. Roman, t. 16, p.687.
} 
hors du champ de la science. Alors que l'actualisation des connaissances était l'un des réquisits les plus pressants associés à l'entreprise encyclopédique, l'Encyclopédie du XIX siècle refuse tout bonnement de faire advenir au statut d'objet [p.191] de science les productions romanesques contemporaines ${ }^{42}$. L'Encyclopédie catholique, en revanche, ne fait pas montre d'une telle retenue et affronte résolument la question des ouvrages fictionnels de son époque. S'opère même un curieux croisement chronologique puisque son article « Roman » commence exactement là où celui de l'Encyclopédie du XIX siècle s'arrête, c'est-àdire à la fin des années 1820. Se refusant à dresser un panorama exhaustif ou à faire l'inventaire des sous-genres existants, l'article de l'Encyclopédie catholique développe d'emblée, et très longuement, la question du « roman moderne en France », c'est-à-dire entre 1830 et 1848. Il analyse avec sévérité la production de trois auteurs (George Sand, Balzac et Sue), dont le succès s'explique, selon lui, par une adéquation particulière du talent avec une des phases sociales troublées, successivement traversées par la France pendant cette période ${ }^{43}$.

Ainsi, alors que l'Encyclopédie $d u X I X^{e}$ siècle refuse de s'engager dans le débat contemporain et s'en tient à une prudente perspective d'histoire littéraire reculée, l'Encyclopédie catholique détaille la production du temps et la met en étroite relation avec l'évolution de la société pour mieux mettre en garde son lecteur contre la démoralisation croissante qui s'exprime par ce genre. Pour l'Encyclopédie catholique, le champ littéraire est un domaine privilégié de la science catholique parce qu'il est un lieu de combat où il [p. 192] y a urgence à agir. D'ailleurs, le zèle apologétique de cette entreprise encyclopédique est particulièrement sensible tout au long de son existence, y compris jusqu'aux trois tomes de son Supplément paru en 1859 : «La foi catholique donc, dans toute son intégrité et dans toute sa rigueur, voilà l'esprit qui fait l'unité de l'ouvrage ${ }^{44}$. Faut-il chercher dans cette

\footnotetext{
42 On peut d'ailleurs faire exactement la même remarque à propos de l'article «Littératures » de cette encyclopédie. La structure de l'article est largement chronologique : elle examine d'abord les littératures grecque et latine, puis la littérature française, depuis les premiers textes en français jusqu'au Moyen Age et à la Renaissance, et s'arrête à la toute fin du XVIII ${ }^{\mathrm{e}}$ siècle. Il faut attendre la troisième édition de l'ouvrage, en 1867, pour que cet article «Littératures", inchangé depuis la première édition, soit suivi d'un article "Littérature française contemporaine », et que soient abordées, et encore de manière très timide, les productions du début du $\mathrm{XIX}^{\mathrm{e}}$ siècle, bien loin en tout cas de la contemporanéité annoncée.

${ }^{43}$ « [...] il y a eu, selon nous, une cause générale qui a fait réussir, dans chaque phase de situation, telle nature de talent plutôt que telle autre [...]. On a vu que le succès du genre inauguré par George Sand coïncidait avec une situation sociale analogue, c'est-à-dire avec les vagues espérances et les utopies indéfinies des rêveurs et des poètes, placés sous l'influence de la chaude atmosphère d'un lendemain de révolution. On a vu que le succès du genre inauguré par M. de Balzac coïncidait également avec une situation sociale amenée par la fuite de ces rêves, par le triomphe du fait sur les idées, et le règne de la force et de la corruption. Il doit en être de même pour le genre inauguré par M. Sue, et il en est de même en effet, le roman de M. Sue est une aspiration vers le socialisme matérialiste. Son roman socialiste est le précurseur de MM. Considérant, Louis Blanc, Cabet et Proudhon » (art. Roman, t. 16, p.685).

${ }^{44}$ «Catholiques avant tout, les collaborateurs n'ont pas à changer la marche suivie dans le corps de l'ouvrage. Leur devise est et doit être: In necessariis unitas, in dubiis libertas, in omnibus charitas. La foi catholique donc, dans toute son intégrité et dans toute sa rigueur, voilà l'esprit qui fait l'unité de l'ouvrage. Dans les choses
} 
intransigeance l'une des raisons de la perte de vitesse ${ }^{45}$ puis de la complète disparition de l'Encyclopédie catholique ? Des circonstances économiques et humaines ne sont sûrement pas à écarter ; néanmoins, si l'on compare l'évolution de l'Encyclopédie catholique avec celle de l'Encyclopédie du XIX siècle, on ne peut s'empêcher de le penser.

En effet, il semble que l'autre titre, vivace jusqu'en 1883, se soit au contraire adapté à l'évolution de son siècle. Dans sa Théorie catholique des sciences, en 1836, Laurentie soulignait clairement l'identité catholique de l'entreprise ${ }^{46}$, et le prospectus de la seconde édition maintenait encore fermement le cap ${ }^{47}$. En revanche, on serait bien en mal de [p. 193] trouver une seule référence explicite au catholicisme dans le péritexte de la troisième édition ; le retournement est même complet. Il ne s'agit plus d'unifier le monde et les connaissances sous le regard de Dieu, mais tout bonnement, et tout profanement, de rendre compte du progrès des connaissances dans toute leur diversité. L'information a changé, ou perdu son sens : à la nécessité d'in-former les connaissances (de les mettre en forme) pour les transmettre au fidèle succède le seul souci d'informer le lecteur (de le tenir au courant) du progrès des connaissances. Cette mutation idéologique profonde est concomitante avec une innovation éditoriale, la parution d'un Annuaire encyclopédique qui, à partir de 1861, inaugure une périodicité régulière et est présenté comme «l'encyclopédie du temps présent, destinée à reproduire, année par année, le mouvement universel de l'activité humaine durant le dernier

douteuses, ils croient devoir exposer avec impartialité les divers systèmes, sans condamner ceux que l'Église n'a pas condamnés; mais ils ne cacheront pas que les doctrines soutenues par les plus grands saints et hautement approuvées par le Saint-Siège apostolique ont toutes leurs préférences, et que, selon eux, c'est l'attachement au Saint-Siège et la soumission à toutes ses décisions qui font la force du catholicisme et qui favorisent le plus la propagation de l'Église parmi les peuples. Ils tendront surtout à conserver en tout la charité, et leur polémique sera toujours grave et sérieuse, comme elle doit l'être dans un ouvrage si important » (Supplément à l'Encyclopédie catholique, Préface de J. Chantrel, t. 1, p.IV).

${ }^{45}$ Le premier tome du Supplément comporte les lettres A à C, le second les lettres C à F, tandis que le troisième survole tout le reste de l'alphabet (G à Z).

46 «L'Encyclopédie du XIX $X^{e}$ siècle, c'est l'ensemble des sciences humaines envisagées sous le point de vue chrétien; ou plutôt c'est l'unité rétablie dans le domaine des connaissances; c'est la dignité rendue à l'intelligence; c'est l'utilité rendue aux études; c'est la perfection même rendue à tous les arts »(PierreSébastien Laurentie, Théorie catholique des sciences, p.3). Point de vue chrétien, c'est-à-dire catholique, bien sûr : «Sous notre plume catholicisme et christianisme, c'est une identité » (ibid., p.84).

${ }^{47}$ «L'Encyclopédie du XIX $X^{e}$ siècle s'est terminée au moment où des faits d'une haute signification lui donnait une importance toute nouvelle. Elle était désignée dans l'encyclique où Sa Sainteté exhortait l'épiscopat et le clergé français «à encourager, de la part des hommes de talent, partisans de la saine doctrine, la publication d'ouvrages propres à éclairer l'esprit de la génération actuelle et à dissiper les erreurs qui tendent à la séduire ». S. E. Mgr l'archevêque de Bordeaux, dans une lettre pastorale, rappelait que l'Église veut la science, recommande l'instruction, sanctionne tout ce qui [la] favorise, condamne tout ce qui l'entrave, et qu'elle est la mère des sciences, parce que Dieu est Père des Lumières. L'Encyclopédie du XIX siècle répond parfaitement à la mission que les circonstances imposent au Clergé. Elle est pour lui comme un arsenal inépuisable où il trouve des armes contre toutes les idées fausses, des arguments contre tous les systèmes subversifs. C'est en un mot le seul ouvrage où le prêtre, outre les questions qui se rattachent aux diverses branches de la science ecclésiastique et aux travaux de ses conférences, soit certain de puiser, en toute sécurité, l'instruction nécessaire à la lutte qui l'attend » (Prospectus de l'éd. de 1862, non paginé ; cité par Claude Bénichou, «L’ignorance des savoirs », La Science catholique..., p.101-102). 
tiers de notre siècle $»^{48}$. Il est le «complément indispensable de l'Encyclopédie du XIX siècle » qui, pour les détails, renvoie à lui comme à un lieu privilégié « où les questions sont traitées, au point de vue de l'actualité, avec une étendue qu'elle [1'Encyclopédie] ne comportait pas. »

À partir de ce moment, l'Encyclopédie du XIX $X^{e}$ siècle se trouve donc dans une situation quelque peu paradoxale : alors qu'elle continue à reproduire dans ses colonnes un article «Roman» qui se termine par un refus clair et net de prendre position sur les productions contemporaines (c'est-à-dire postérieures au début du siècle.... $)^{49}$, elle est dorénavant accompagnée d'un Annuaire qui, lui, dès la première livraison, dessine un ample panorama de la littérature française des [p. 194] derniers mois ${ }^{50}$. Au prix de cette curiosité, l'Encyclopédie du $X I X^{e}$ siècle peut s'inscrire dans la durée, voire dans l'éternité, comme le souligne l'Avertissement de l'Annuaire de 1865-1866 en annonçant la parution d'une «troisième et dernière édition » de l'Encyclopédie :

Cette œuvre monumentale aura le privilège de ne plus vieillir désormais : maintenue par l'Annuaire encyclopédique au courant de tous les événements, de toutes les inventions, de toutes les découvertes et de tous les progrès, elle restera la vivante expression du siècle dont elle porte le nom.

Certes, la modération et le respect des conventions intellectuelles et sociales ont toujours été plus de mise dans l'Encyclopédie du XIX siècle ${ }^{51}$ que dans l'Encyclopédie catholique. Mais ce qui explique surtout l'évolution divergente des deux titres est la composition du personnel qui les rédige depuis leurs origines. Cuvier et Ampère ont été les fondateurs de l'Encyclopédie $d u X I X^{e}$ siècle, et l'ouvrage a été continué «sous la direction vigilante d'un comité de savants, choisis dans les diverses classes de 1'Institut $»^{52}$. On relève ainsi au bas des articles les noms de Arago, Champollion, Geoffroy Saint-Hilaire, d'Orbigny ou Royer-Collard. En face, à l'Encyclopédie catholique, les noms sont nettement moins prestigieux et le choix des collaborateurs, du moins pour les articles les moins idéologiquement exposés, est beaucoup plus éclectique (ce qui explique la surprenante

\footnotetext{
${ }^{48}$ Encyclopédie du XIX ${ }^{e}$ siècle, $3^{\mathrm{e}}$ éd., Avertissement, 1867, t. 1, p.VII.

${ }^{49}$ L'édition de 1867 comporte un article «Littérature française contemporaine » (t. 13, p.792-800, signé par Jules Levallois) qui ne change rien au problème : il traite essentiellement du XVIII ${ }^{\mathrm{e}}$ siècle et ne fait qu'effleurer le XIX ${ }^{\mathrm{e}}$.

${ }^{50}$ Annuaire encyclopédique. Politique, économie sociale, statistique, administration, sciences, littérature, beauxarts, agriculture, commerce, industrie, 1859-1860, Paris, Au bureau de l'Encyclopédie du XIX ${ }^{\mathrm{e}}$ siècle, 1861, p.708-721.

${ }^{51}$ Ainsi, il est « défendu [à une encyclopédie] d'entrer dans l'arène des controverses flagrantes, de se mêler aux débats contemporains, d'adopter ou de combattre les frivolités et les passions qui circulent autour d'elle » (art. Encyclopédie, 1867 ( $3^{\mathrm{e}}$ éd.), t. 9, p.541).

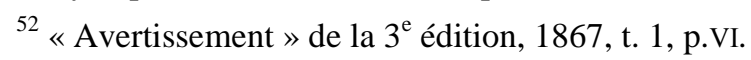


présence, toute alimentaire, précisons-le, de Proudhon ${ }^{53}$ au nombre des rédacteurs). En revanche, l'essentiel des articles à portée idéologique forte est confié à des personnages dont l'orthodoxie est inflexible et ne s'accompagne que très rarement d'une reconnaissance institutionnelle. Ces rédacteurs sont essentiellement recrutés dans le corps professoral des séminaires (comme l'abbé Glaire) ou des institutions propres à l'Église catholique (l'abbé Guérin, très actif dans le Supplément est «membre de l'Académie catholique [p. 195] de Rome »), chez des ecclésiastiques inconnus du grand public, ou surtout dans la cohorte des polygraphes et autres publicistes qui peuplent les nombreux journaux et revues catholiques. C'est le cas du promoteur de l'entreprise, le vicomte Joseph-Alexis Walsh (1782-1860), ou de Joseph Chantrel (1818-1884), maître d'œuvre et rédacteur de la préface du Supplément.

L'opposition entre les deux modes de recrutement est peut-être encore plus visible pour les articles du champ littéraire. Dans le cas de l'Encyclopédie du XIX $X^{e}$ siècle, les rédacteurs sont presque tous des universitaires, bardés de diplômes et occupant des places de premier rang dans l'institution. Parmi eux, Philarète Chasles (1798-1873), professeur au Collège de France, et Eugène Geruzez (1799-1865), professeur à la Faculté des lettres de Paris et maître de conférence à l'École normale supérieure, jouent un rôle de premier plan. À l'opposé, Alfred Nettement (1805-1869) est «la » plume littéraire de 1'Encyclopédie catholique. En dépit des très nombreux ouvrages de critique littéraire qu'il a publiés et dont de larges extraits alimentent les colonnes de l'Encyclopédie catholique, il ne peut se prévaloir d'aucune légitimité institutionnelle, ce qui explique vraisemblablement pour une part l'aménité pour le moins mesurée du jugement qu'il porte sur son «collègue » Chasles dans son Histoire de la littérature française ${ }^{54}$.

La floraison des entreprises encyclopédiques catholiques dans la première moitié du $\mathrm{XIX}^{\mathrm{e}}$ siècle résulte donc d'une brève conjoncture favorable pendant laquelle des intellectuels catholiques ont pensé que la foi et la science pouvaient - de nouveau - marcher d'un même pas, et ont souhaité revenir sur l'exclusive prononcée par les Lumières. Mais l'état de grâce n'a pas duré. Dès le deuxième tiers du siècle, il est clairement apparu que la propagande par

\footnotetext{
${ }^{53}$ Sur les 29 articles rédigés par Proudhon pour le t. 2 de l'Encyclopédie catholique, voir l'introduction de Théodore Ruyssen aux Euvres complètes. Nouvelle édition. Écrits sur la Religion, Paris, Marcel Rivière, 1959, p.28 et p.90.

54 «M. Philarète Chasles, qui appartient à la même famille de critiques que M. Saint-Marc Girardin, ne paraît pas se préoccuper beaucoup plus que lui du besoin de remonter à des idées générales en littérature. L'action qu'il exerce consiste surtout à initier le lecteur français à la connaissance profonde et vraie des littératures et des sociétés étrangères. [...] Il est favorable aux idées littéraires nouvelles, mais il évite les excès auxquels plusieurs écrivains de l'école romantique de son temps se laissent emporter, ce qui ne l'empêche point d'avoir un sentiment juste et élevé de la poétique du Moyen Age » (Histoire de la littérature française. II, Sous le Gouvernement de Juillet, Paris, Jacques Lecoffre, 1854, t. 1, p.103-104).
} 
les bons livres, caractéristique de l'entreprise de Migne et irriguant pour une part les projets des deux encyclopédies de forme plus classique, n'est plus [p. 196] adaptée au développement des sciences et à la réception du progrès par les fidèles cultivés. Ce public n'adhère plus à un projet forçant la science à entrer dans le cadre rigide d'un programme apologétique qui, même pour les catholiques, ne peut plus être présenté en ces termes. Enfin, le temps passant, la polémique exacerbée contre l'Encyclopédie de Diderot et d'Alembert commence à faire long feu. Il faut alors s'adapter, comme l'Encyclopédie du XIX siècle, ou disparaître, comme l'Encyclopédie catholique. 